\title{
In vitro reassembly of nuclear envelopes and organelles in Xenopus egg extracts
}

\author{
Ping Lu ${ }^{1}$, Hui Zheng ${ }^{2}$, Zhonghe Zhai ${ }^{1}$ \\ ${ }^{1}$ College of Life Sciences; ${ }^{2}$ Institute of Molecular Medicine, Peking University, Beijing 100871, China
}

\begin{abstract}
We reconstituted bilayer nuclear membranes, multilayer membranes, and organelles from mixtures of Xenopus laevis egg extracts and demembranated Xenopus sperm nuclei. Varying proportions of the cytosolic and vesicular fractions from the eggs were used in the reconstitution mixtures. A cytosol:vesicle ratio of 10:1 promoted reassembly of the normal bilayer nuclear membrane with inserted nuclear pore complexes around the decondensed Xenopus sperm chromatin. A cytosol: vesicle ratio of 5:1 caused decondensed and dispersed sperm chromatin to be either surrounded by or divided by unusual multilayer membrane structures with inlaid pore complexes. A cytosol:vesicle ratio of 2.5:1 promoted reconstitution of mitochondria, endoplasmic reticulum networks, and Golgi apparatus. During reassembly of the endoplasmic reticulum and Golgi apparatus, vesicular fragments of the corresponding organelles fused together and changed their shape to form flattened cisternae, which were then stacked one on top of another.
\end{abstract}

Cell Research (2006) 16:632-640. doi: 10.1038/sj.cr.7310066; published online 30 May 2006

Keywords: cell-free system, endoplasmic reticulum, Golgi apparatus, mitochondria, nuclear reconstitution, Xenopus egg extracts

\section{Introduction}

During mitosis, a cell needs to partition its duplicated genetic material into two daughter cells under very strict control in a program that comprises several checkpoints. Equally accurate partitioning of the intracellular organelles that sustain vital cellular function is also required [1]. At the onset of mitosis (or meiosis), the nuclear envelope breaks down, as do the endoplasmic reticulum, the Golgi apparatus, and the mitochondria. At telephase, all of the organelles are rapidly reassembled from their fragments, which are present in each daughter cell. Membranes of most cellular organelles, including the nuclear envelope, the endoplasmic reticulum and Golgi cisternae, and the mitochondrial cristae, have complicated shapes in which two membranes are closely apposed to one another. How

Correspondence: Ping Lu

Tel: +86-10-62754050; Fax: +86-10-62751526;

E-mail: luping@pku.edu.cn

Received 8 Oct 2005; revised 24 Feb 2006; accepted 20 Mar 2006; published online 30 May 2006 these structures are generated and maintained is largely unknown [2].

Although organelle disassembly and reassembly has been the focus of considerable research, an integrated picture of the way in which components interact to form a functioning cell is still lacking. The two principal obstacles to research in this field are the complexity of the mechanisms of cell division and proliferation, and the absence of techniques for tracing and controlling the process of cell division in vivo.

Cell-free systems have proven to be powerful tools for understanding the molecular basis of cellular function. Because cell-free preparations are amenable to a wider range of manipulation than are intact cells, they are particularly useful in biochemical investigations of the cell cycle. It has been shown that in vitro nuclear assembly spontaneously in Xenopus egg interphase extracts a double nuclear membrane with nuclear pore forms around added chromatin, whether natural animal or plant sperm chromatin or exogenously added procaryotic DNA is used [3-5]. Some information concerning reassembly of nuclear membranes, organelles, and individual nucleosomes has 
arisen from the use of cell-free extracts derived from eggs of Xenopus or sea urchin, and somatic cells and embryos of Drosophila or Nicotiana [2, 6-14].

The Golgi apparatus is one of the most complex intracellular organelles. Its basic units are disc-shaped cisternae, each of which features a flattened central portion that is closely apposed to adjacent cisternae in a stack. Each face of the stack is apposed to an extensive tubular reticulum termed the cis- and trans-Golgi network [15]. In mammalian cells, four to eight cisternae are stacked in a polarized manner, and numerous such stacks are connected [16]. The Golgi apparatus is disassembled at the onset of mitosis and reassembled at the end of mitosis. The disassembly-reassembly process is generally believed to be essential for equal partitioning of the Golgi apparatus into daughter cells [17]. During mitosis, the Golgi apparatus fragments into tens of thousands of vesicles and short tubules that disperse throughout the cytoplasm. The number and random dispersion of these fragments ensures accurate partitioning. At telephase, a Golgi apparatus is rapidly reassembled from its fragments within each daughter cell.

The endoplasmic reticulum is a structure within eucaryotic cells that is highly variable in shape. Sometimes it exists as a continuum, and sometimes it may be observed as a number of isolated components that can fuse to form a continuum. Structural modifications of the ER, including dilation-vesiculation of rough cisternae, as well as smooth tubule proliferation can also coincide with changes in cytoskeleton organization and changes in cell cycle [7]. At the end of mitosis, membranes are recruited to the chromatin, probably in the form of ER sheets, and fuse side-to-side, encompassing the DNA with an intact double nuclear membrane [18].

Each Xenopus egg contains a stockpile of all components of the nucleus and the cytoplasm, including histones, lamins, pore complex components, and membrane vesicles, sufficient to assemble more than 4000 somatic nuclei. These nuclei are derived from the rapid division of zygote 12 times, which take place in the early embryo [19]. The endoplasmic reticulum, Golgi apparatus, and mitochondria of fertilized ova are present in small membrane tubules and vesicles that, at some point during early development, must fuse to reform the endoplasmic reticulum network, Golgi apparatus, and mitochondria. The machinery for the formation of these structures must also be stored in the egg because there is no synthesis of new material during the first cell divisions [2]. From a biochemical point of view, fragmentation of the cell structures allows their homogenization, with the added advantage that it occurs in such a way as to permit reassembly of the original organelle. If it was possible to mimic reassembly of these cellular structures using a cellular homogenate in vitro, identification of the components underlying organelle structure would be greatly facilitated [20]. Xenopus egg extracts provide an extraordinary opportunity for us to achieve this aim.

In the present report, we describe the formation of bilayer or multilayer nuclear envelopes in vitro using Xenopus egg extracts. This cell-free system was also used to successfully reconstitute many kinds of organelles, including mitochondria, endoplasmic reticulum networks, and Golgi apparatus, by varying the cytosol:vesicle ratio in the reconstitution mixture. Of particular interest is the fact that this report is the first published account of the successful reassembly of mitochondria in vitro.

\section{Materials and methods}

\section{Preparation of egg extracts}

The following protocol was modified from Newport and Spamm [21] and Sider et al. [22]. Mature Xenopus females were induced to lay eggs by injections of HCG (human chlorionic gonadotrophin) the night before use and were placed in $3 \mathrm{~L}$ of $110 \mathrm{mM} \mathrm{NaCl}$. The eggs were degelled with three egg volumes of $2 \%$ cysteine $(\mathrm{pH}$ 7.8) dissolved in $110 \mathrm{mM} \mathrm{NaCl}$ for 5-10 min until the eggs were closely packed. The degelled eggs were rinsed three times with MMR (0.1 mM NaCl, $2 \mathrm{mM} \mathrm{KCl,} 1 \mathrm{mM} \mathrm{MgSO}$, $2 \mathrm{mM} \mathrm{CaCl}_{2}$, $0.1 \mathrm{mM}$ ethylenediaminetetraacetic acid, $5 \mathrm{mM} \mathrm{N}$-(2-hydroxyethyl)piperazine- $N^{\prime}$-(2-ethanesulfonic acid), $\mathrm{pH} 7.8$ ) to remove cysteine. They were then rinsed twice in S-lysis buffer $[250 \mathrm{mM}$ sucrose, $2.5 \mathrm{mM} \mathrm{MgCl} 2,50 \mathrm{mM} \mathrm{KCl}, 100 \mu \mathrm{g} / \mathrm{mL}$ cycloheximide, $5 \mu \mathrm{g} / \mathrm{mL}$ cytochalasin $\mathrm{B}, 1 \mathrm{mM}$ dithiothreitol (DDT), $0.1 \mathrm{mM}$ phenylmethysulfonyl fluoride, $\mathrm{pH}$ 7.5)]. All centrifugation steps were conducted at $4{ }^{\circ} \mathrm{C}$. The eggs were packed by centrifugation at 100 $\times g$ for $30 \mathrm{~s}$, and excess S-lysis buffer was removed thoroughly. The eggs were then crushed by centrifugation at $15000 \times g$ for $30 \mathrm{~min}$. As a result, the egg components were distributed in the centrifuge tube with the yolk at the bottom, the crude extract in the middle, and a lipid layer at the top. The crude extract was removed, placed into a new tube, and centrifuged again for $15 \mathrm{~min}$ to remove the remaining contaminating yolk and large particulate material. The crude extract was then centrifuged at $200000 \times g$ for $2 \mathrm{~h}$ using a TLS-55 swingingbucket rotor at $55000 \mathrm{rpm}$ in a Beckman Optima TL and TLX. After ultracentrifugation, the crude extracts were laminated into seven layers. These layers (from the top to the bottom of the tube) were lipid, cytosol (egg extracts), membrane vesicles, mitochondria, ribosomes, glycogen, yolk, and pigment granules. The cytosol layer was carefully transferred into a new tube and centrifuged again at $200000 \times g$ for $20 \mathrm{~min}$ to remove residual membrane vesicles. The membrane vesicle layer was resuspended in S-lysis buffer containing $0.5 \mathrm{M}$ sucrose and centrifuged at $26000 \times \mathrm{g}$ for $15 \mathrm{~min}$ to pellet cytosol-depleted membrane vesicles. The cytosolic and membrane vesicle fractions were quick-frozen in liquid nitrogen as $50 \mu \mathrm{L}$ and $5 \mu \mathrm{L}$ aliquots, respectively, and stored at $-70{ }^{\circ} \mathrm{C}$.

\section{Preparation of sperm nuclei}

Testes were dissected from sexually mature Xenopus laevis males and rinsed free of blood. Sperm were released by gently squeezing the 
testes in $1 \mathrm{~mL}$ of nuclear isolation buffer (NIB) consisting of $15 \mathrm{mM}$ $\mathrm{NaCl}, 60 \mathrm{mM} \mathrm{KCl}, 15 \mathrm{mM}$ Tris, $1 \mathrm{mM}$ DDT, $0.5 \mathrm{mM}$ spermin, 0.25 $\mathrm{M}$ sucrose, $\mathrm{pH}$ 7.5. Somatic tissue was removed by centrifugation at $100 \times g$ for $1 \mathrm{~min}$ at $4{ }^{\circ} \mathrm{C}$. The supernatant fraction containing sperm was then centrifuged at $1500 \times g$ for $10 \mathrm{~min}$ at $4{ }^{\circ} \mathrm{C}$. We slowly resuspended the white part of the sediment in NIB, carefully avoiding the red cell pellet. Sperm were collected by centrifugation and incubated at $22{ }^{\circ} \mathrm{C}$ in $1 \mathrm{~mL}$ of NIB containing $0.05 \%$ lysolecithin for 8-10 min, at the time when three volumes of cold NIB containing $3 \%$ bovine serum albumin were added to the sperm suspension to stop the lysolecithin reaction. Nuclei were then washed three times in NIB. The lysolecithin treatment removed the sperm plasma membrane and nuclear envelope, while leaving the chromatin in its highly condensed state. The density of the demembranated sperm nuclei was adjusted to $4 \times 10^{7} / \mathrm{mL}$, and the suspension was quick-frozen in liquid nitrogen in $5-\mu \mathrm{L}$ aliquots and stored at $-70{ }^{\circ} \mathrm{C}$.

\section{Nuclear reassembly}

Demembranated Xenopus sperm $(5 \mu \mathrm{L})$ was mixed with $45 \mu \mathrm{L}$ of a mixture containing Xenopus egg extract and membrane vesicles in an ATP-regenerating system containing $2 \mathrm{mM}$ ATP, $20 \mathrm{mM}$ phosphocreatine, and $50 \mu \mathrm{g} / \mathrm{mL}$ creatine phosphokinase. The mixture was incubated at $22^{\circ} \mathrm{C}$. Three different ratios of Xenopus egg cytosol to membrane vesicle fractions were used: 10:1, 5:1, and 2.5:1.

\section{Fluorescence microscopy}

At 15-30-min intervals, a $2-\mu \mathrm{L}$ aliquot of the nuclear reconstitution mixture was placed on a slide containing $2 \mu \mathrm{L}$ of $2.5 \%$ glutaraldehyde and $1 \mu \mathrm{L}$ of $5 \mu \mathrm{g} / \mathrm{mL}$ of the fluorescent DNA dye 4, 6-diamidine2 phenylindole. The process of sperm pronuclear assembly was observed using a Leica fluorescence microscope.

\section{Transmission electron microscopy}

At $30-\mathrm{min}$ intervals, a $10-\mu \mathrm{L}$ aliquot of the nuclear reassembly reaction was placed in a tube containing $200 \mu \mathrm{L}$ of $2.5 \%$ glutaraldehyde, and incubated for $2 \mathrm{~h}$ at $4{ }^{\circ} \mathrm{C}$. Samples were washed three times with phosphate buffer ( $\mathrm{pH} 7.4$ ), and post-fixed for $2 \mathrm{~h}$ in $1 \%$ osmium tetroxide at $4{ }^{\circ} \mathrm{C}$. The fixed mixtures were dehydrated using a graded concentration series of acetone, and embedded in Epon 812. Sections were made at 50-60 nm and stained with uranyl acetate followed by lead citrate. The samples were observed and photographed at $80 \mathrm{kV}$ under a JEM 1010 transmission electron microscope.

\section{Results}

Nuclear reassembly at a 10:1 ratio of cytosol to vesicle fractions

When observed using fluorescence microscope, demembranated Xenopus sperm (Figure 1A) were found to undergo a series of morphological changes during incubation with Xenopus egg extract mixture. The sperm began to expand after 15-30 min of incubation with the egg extract mixture, and they became plumper and shorter than their original long, tadpole-like shape (Figure 1B and 1C). After an additional 15-30 min of incubation, the sperm DNA started to disperse (Figure 1D and 1E). After $1.5 \mathrm{~h}$ of incubation, the sperm DNA was completely decondensed and dispersed, and the sperm became spherical (Figure 1F).

When observed using transmission electron microscope, demembranated Xenopus sperm were found to be long and to have densely packed chromatin (Figure 2A). After 30 min of incubation, the demembranated sperm swelled and shortened in length (Figure 2B). The membrane vesicles started to recruit to the rim of the swollen demembranated Xenopus sperm. After $60 \mathrm{~min}$ of incubation, the sperm became elliptical or spherical, and the bilayer nuclear envelope was partially assembled (arrowheads in Figure 2C). A pair of vesicles about to fuse was observed (arrow in Figure 2C). After incubation for $1.5 \mathrm{~h}$, the reassembled nucleus was observed with double nuclear membrane inserted nuclear pore complexes (Figure 2D). One of the nuclear pore complexes reconstituted was enlarged and is shown (arrow in Figure 2D-1) (Figure 2D-1 was the magnifying figure of right-side nuclear pore complex in Figure 2D). A cytosol:vesicle ratio of 10:1 was optimal for the formation of a normal bilayer membrane containing nuclear pore complex.

\section{Nuclear reassembly at a 5:1 ratio of cytosol to vesicle fractions}

Observation by transmission electron microscope showed that the nuclear reassembly process of the
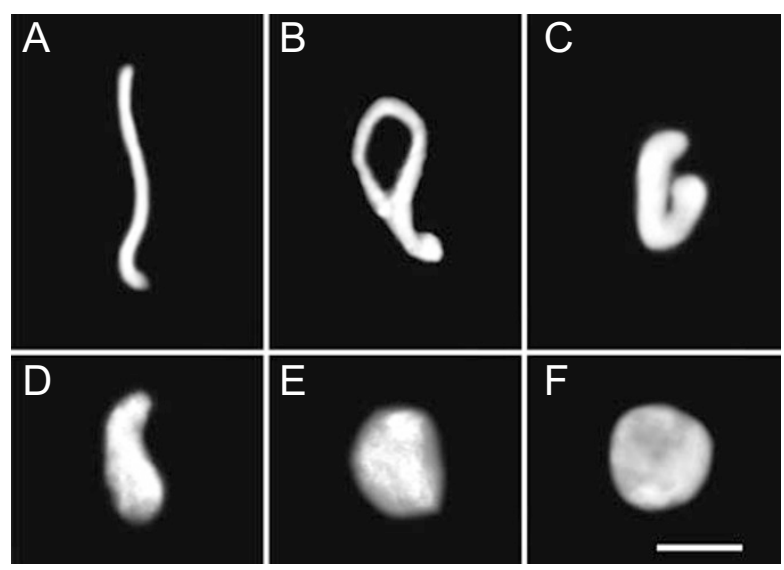

Figure 1 Nuclear reconstitution from demembranated $X$. laevis sperm in Xenopus egg extracts visualized using fluorescence microscope. The egg cytosol:vesicle ratio in the reconstitution mixture was 10:1. (A) Demembranated sperm. (B) Demembranated sperm after a 15-min incubation with egg extract. (C) Swollen sperm (30-min incubation). (D) Expanded and dispersed sperm (45-min incubation). (E) Decondensed sperm chromatin (60-min incubation). (F) Completely decondensed chromatin in spherical sperm (90-min incubation). Bar $=10 \mu \mathrm{m}$. 


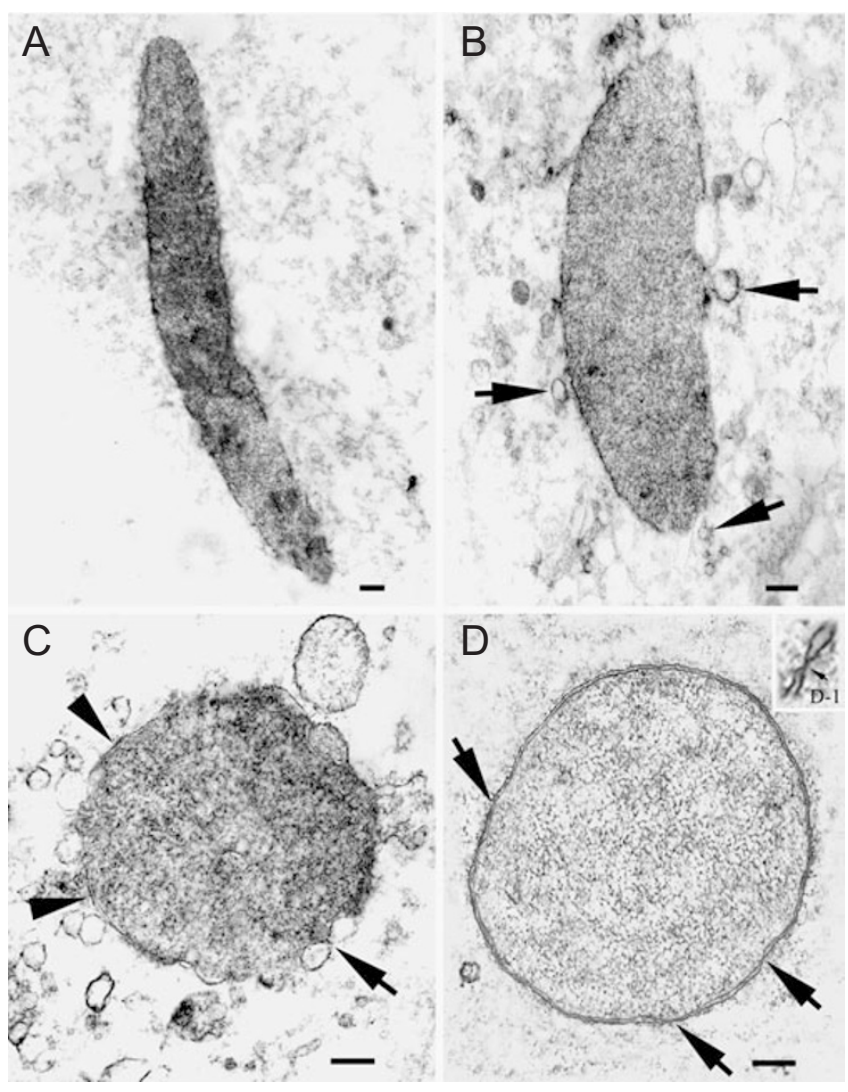

Figure 2 Nuclear reconstitution from demembranated sperm in egg extracts visualized using transmission electron microscope. The egg cytosol:vesicle ratio in the reconstitution mixture was 10:1. (A) Demembranated sperm. (B) After a 30-min incubation with egg extract, the sperm began to swell, and membrane vesicles (arrowhead) were recruited to the sperm edge. (C) After a 60-min incubation, the sperm continued to decondense, and became spherical. A bilayer nuclear membrane (arrowhead) was partially assembled at the sperm periphery. The arrow indicates a pair of fusing vesicles. (D) The reassembled nucleus has a double envelope with inserted nuclear pore complexes (arrows). (D-1) A nuclear pore complex enlarged from right-side nuclear pore complex (D, arrow). Bar $=1 \mu \mathrm{m}$.

demembranated Xenopus sperm in a mixture composed of a 5:1 ratio of egg cytosolic to vesicular fractions was quite different from that described above for a 10:1 ratio. After incubation for $30 \mathrm{~min}$, a nuclear reassembly intermediate was observed. This intermediate had dispersed chromatin, and was surrounded by many vesicles (Figure 3A). After incubation for $1 \mathrm{~h}$, another, more highly organized intermediate appeared. This intermediate was surrounded by a membrane (arrow) and contained two small, reassembled "nuclei" (arrowheads in Figure 3B). After $1.5 \mathrm{~h}$ of incubation, many unusual structures appeared (Figure 3C and 3D). These structures usually consisted of multilayer membranes with inserted nuclear pore complexes (arrows in Figure 3C and 3D, and Figure 3C1 and 3D-1 were, respectively, the section-magnifying figures of Figure 3C and 3D). Sometimes, a bilayer or multilayer membrane separated the chromatin into different areas within the unusual nucleus-like structures (Figure $3 \mathrm{C}$ and $3 \mathrm{D})$.

Reassembly of endoplasmic reticulum, mitochondria, and Golgi apparatus at a 2.5:1 ratio of cytosol to vesicle fractions

For the demembranated sperm mixture incubated with an egg cytosol:vesicle ratio of 2.5:1, we observed reconstitution of organelles other than nuclei (endoplasmic reticulum network, Golgi apparatus, and mitochondria). Most of the demembranated sperm chromatin remained condensed after $2 \mathrm{~h}$ of incubation (Figure 4A). We did not observe pronucleus-like structures with reassembled nuclear membranes under transmission electron microscope in the 2.5:1 mixture.

Reassembling endoplasmic reticulum structures were observed after 30-60 min of incubation (Figure 4B and $4 \mathrm{C})$. The endoplasmic reticulum reassembly process appeared to be the reverse of the disassembly process. First, the fragmented endoplasmic reticulum vesicles grouped together, and two or more vesicles fused together $(1,2$ in Figure 4B). This event was followed by a morphological change to form short cisterna structures. A larger vesicle arising from the fusion of smaller vesicles was observed to be simultaneously changing the shape to form a cisterna and fusing with another vesicle (3 in Figure 4B). These short cisterna structures fused to produce longer and more flattened cisternae, and overlapped each other to form layered stacks of cisternae. An endoplasmic reticulum that is reassembling after $30 \mathrm{~min}$ of incubation is shown in Figure 4B. Two sets of stacked cisternae in the process of maturing are shown in Figure 4B-4 and 4B-5. Their appearance is that of ribbon-like structures stacked together. In the assembling endoplasmic reticulum, many ribosome particles are visible in the newly formed cisternae (arrows in Figure 4B). Most of the vesicles that fused to form the endoplasmic reticulum were similar and carried many ribosomes (arrowheads in Figure 4B). The sectionmagnifying picture of Figure 4B shows ribosomes clearly (arrows in Figure 4B-1). In addition, we discovered that when the proportion of egg cytosol in the reassembly mixture was low, the vesicles fused to form larger vesicular structures rather than the more highly organized cisternal structures (6 in Figure 4B).

A mature, artificially reconstituted endoplasmic reticulum that formed after $1 \mathrm{~h}$ of incubation is shown in Figure 4C. It has a complex network structure, as well as 

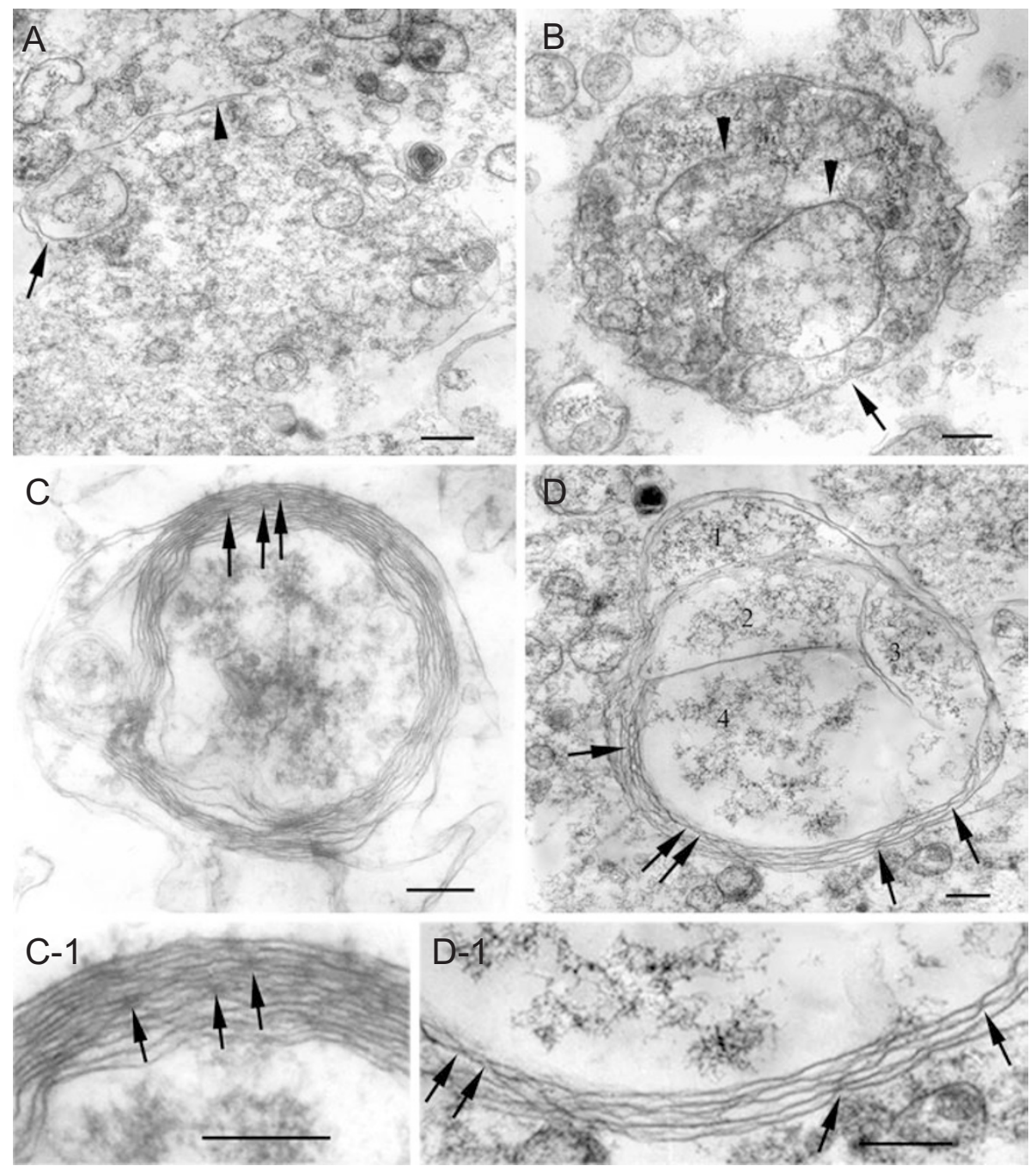

Figure 3 Nuclear reconstitution from demembranated sperm in egg extracts visualized using transmission electron microscope. The egg cytosol:vesicle ratio in the reconstitution mixture was 5:1. (A) Demembranated Xenopus sperm after a 30-min incubation with egg extract. Many vesicles were recruited to the decondensed Xenopus sperm, and some vesicles fused to form larger vesicles (arrow). The membrane structure was partially reassembled (arrows). (B) A reconstitution intermediate composed of two reassembled "nuclei" (arrows) surrounded by many vesicles, and embedded within a larger, reconstituting nucleus (arrow; 60-min incubation). (C) The reassembled nucleus has a multilayer concentric membrane with inserted nuclear pore complexes (arrowheads; 90-min incubation). (C-1) The magnifying figure of section with nuclear pore complexes in (C, arrow). (D) A reconstituted nucleus divided into four parts (1-4) by a reassembled membrane decorated with nuclear pore complexes (arrows; 90-min incubation). (D-1) The magnifying figure of section with nuclear pore complexes in (D, arrow). Bar=1 $\mu \mathrm{m}$.

cisternal junctions. Because sufficient time was elapsed for the reassembly reaction to be complete, junctions (arrow in Figure 4C) between the flattened cisternae had formed.

We observed the reassembly of mitochondrial structures in our cell-free system when the egg cytosol: vesicle ratio was 2.5:1. In Figure $4 \mathrm{D}$, a reassembled mitochondrion (arrowhead) is shown near another that is ready for reassembly (arrow). We presumed that the latter mitochondrion would complete the assembly process if more vesicles and incubation time were provided. The cristae of the reassembled mitochondrion were well defined and transparent.

We also observed the reassembly of a Golgi apparatus in the 2.5:1 mixture (Figure 4E). Six cisternae were formed and stacked in a polarized manner. Abutting each side of the stacks was a tubular/vesicular network, the cis- and 


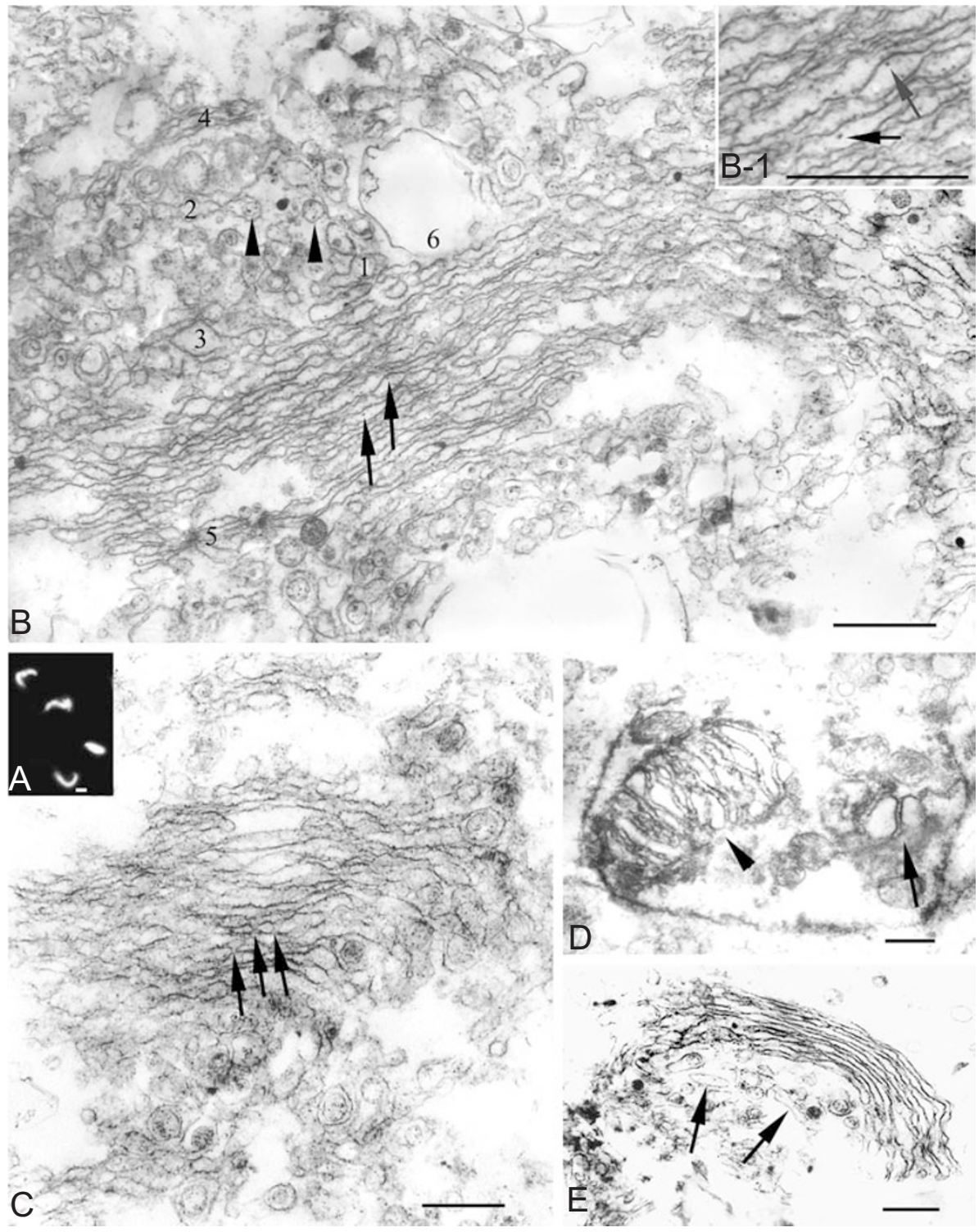

Figure 4 Reconstitution ER, Golgi apparatus, and mitochondria in egg extracts visualized using transmission electron microscope. The egg cytosol:vesicle ratio in the reconstitution mixture was 2.5:1. (A) Most of the demembranated sperm chromatin remained condensed after a 2-h incubation with egg extract. (B) The endoplasmic reticulum reassembly process. Many ribosomes are present in the reassembled cisternae (arrowheads). Most of the vesicles were of the same type, and carried ribosome particles (arrow). 1-6 in (C) show as follows: 1, a pair of vesicles fusing; 2, three vesicles fusing; 3, a larger fused vesicle in the process of simultaneously changing to a flattened shape with a longer cisterna and fusing with another vesicle; 4 and 5, two sets of stacked cisternae being processed; 6, a larger vesicles arising from the fusion of small vesicles. (B-1) Section-magnifying picture of (B) shows ribosomes clearly (arrows). (C) A reassembled endoplasmic reticulum. The arrows indicate the junctions formed between endoplasmic reticulum cisternae (1-h incubation). (D) A reassembled mitochondrion (arrowhead) with well-defined, transparent cristae. A second, partially reassembled mitochondrion (arrow) presumably could finish assembly if sufficient time, space, and vesicles were provided. (E) A reconstituted Golgi apparatus with six cisternae stacked in a polarized manner. Bar $=1 \mu \mathrm{m}$.

trans-Golgi networks. The Golgi cisternae were derived from vesicular fusion followed by a change in shape (arrows in Figure 4E). This process was similar to that of ER reassembly (Figure 4B).

\section{Discussion}

The technological method to extract cytosol and membrane vesicles from Xenopus eggs has 
assured the elimination of all organelles because of the ultracentrifugation at $200000 \times g$ for $2 \mathrm{~h}$ and the breakdown of membrane system triggered by free $\mathrm{Ca}^{2+}$ release as a result of egg rupture [23]. Meanwhile, the cytosol and membrane vesicles were checked carefully with transmission electron microscope. No residual organelles were observed in the two parts. The organelles, that are observed in the paper, were derived from the in vitro reconstitution in cell-free system.

The endoplasmic reticulum, Golgi apparatus, and mitochondrion reconstituted in vitro have similar features of structures with the same kinds of organelles from intact Xenopus eggs and other cells according to morphological characteristics [24-26]. The identifications of organelle structures reconstituted in the paper are tentative and based only on apparent morphological similarity.

The observations of organelle reassembly in Xenopus egg extracts described in this report suggest that the reassembly of the endoplasmic reticulum and Golgi apparatus is essentially the reverse of the disassembly process that has been elucidated by research in vivo. The endoplasmic reticulum and Golgi apparatus appear to have similar reconstitution mechanisms. During the Golgi apparatus and endoplasmic reticulum reassembly processes, only few vesicles from the fragmented organelles first fuse with each other and change their shape to form cisternae (Figure 4B and 4E). These cisternae then stack on top of one another to reconstitute the complex structure of their parent organelles.

In general, repeated fusion of membrane vesicles may be expected to form progressively larger vesicles. Instead, the end products of fusion and assembly are flattened cisternae that are morphologically distinct from the starting material. How does the structure of the flattened cisternae arise? The membrane vesicles may first fuse to form large vesicular structures. These large vesicles then undergo a morphological change, becoming more tubular, and then condense or flatten during the stacking process. Some products of vesicular fusion change their shape to the cisterna shape after the fusion event ( 3 in Figure 4B), and some of them change their shape at the time of fusion, without time intermission (1 and 2 in Figure 4B).

At the onset of mitosis, the Golgi ribbon is broken down into discrete stacks that undergo fragmentation to yield clusters of vesicles (50-70 $\mathrm{nm}$ diameter), large vesicles and tubules (150-250 nm diameter), and cisternal remnants $[1,27]$. Disassembly of the Golgi apparatus occurs in two steps, the first of which generates discrete stacks [28]. In the second step, the stacks fragment to yield small vesicles and short tubules, and eventually free vesicles are distributed at random throughout the cytoplasm of the mitotic cell. Up to 10000 Golgi vesicles are produced, which would, theoretically, and in a stochastic manner, provide almost every daughter cell with $50 \% \pm 1.5 \%$ of the original Golgi membrane $[29,30]$. The proteins that determine the size and shape of the organelles are unknown, in part because biochemical dissection of the process is always difficult.

Similarly, we conclude that vesicles carrying ribosomes (arrows in Figure 4Band 4C) fuse to reconstitute the rough endoplasmic reticulum. However, the force driving the grouping together of huge populations of endoplasmic reticulum vesicles (Figure 4B) and the mechanisms employed for endoplasmic reticulum reassembling remain unknown.

How are the endoplasmic reticulum and Golgi membrane structures generated? A seemingly important clue comes from the fact that in the absence of cytosol, the membranes can fuse, but they form large, spherical vesicles rather than networks (6 in Figure 4B). Furthermore, cytosol must be present during the fusion reaction to mediate tubular network formation. Specific components of the cytosol may thus be required for the vesicle shape change. Thermodynamically, spheres are probably the most stable result of fusion; therefore, one or more cytosolic factors may modify this default reaction to convert it into a fusion reaction that results in a network [2]. In contrast, a low cytosol:vesicle ratio is unfavorable to the formation of the normal bilayer nuclear envelope, instead of the multilayer membrane or multi-double membrane (Figure 3C and 3D).

In our experiments, cytosol was necessary for the reassembly of complex organelle structures, probably because it contains proteins that are involved in vesicle fusion and shape change. High cytosol:vesicle ratios (10:1) were unfavorable to reassembly of organelles other than nuclei, however. One possible explanation for this is that the vesicles may not be at a high enough concentration. Since endoplasmic reticulum networks, Golgi complexes, and mitochondria are composed principally of vesicle components, a certain minimal concentration of vesicles may be essential for the assembly of organelles. Another possible explanation is that a specific balance of cytosolic and vesicular components may be required.

Pharmacological studies indicate that calmodulin is required for late-stage vacuole fusion in vitro and in vivo [31]. A large proportion of the bound calmodulin is released from the vacuole at $\mathrm{Ca}^{2+}$ concentrations below $500 \mathrm{nM}$, and the $\mathrm{Ca}^{2+}$ concentration in the cytosol of living yeast cells is $100-150 \mathrm{nM}$ [32], which is within this range. Released calmodulin can be recovered from the supernatant. Freshly isolated vacuoles (in the presence of $3 \mu \mathrm{M} \mathrm{Ca}^{2+}$ ) carry about $0.15 \mathrm{ng}$ calmodulin per microgram vacuolar protein, corresponding to 20-100 molecules per vacuole [31]. Sullivan et al. [33] consider that calcium mobilization 
is required for nuclear vesicle fusion. An elevated free calcium wave follows fertilization in eggs [34].

Research with various $\mathrm{Ca}^{2+}$ chelators suggests that $\mathrm{Ca}^{2+}$ fluctuations of $\sim 1 \mu \mathrm{M}$ may be important for endoplasmic reticulum network formation. A chelator with a binding constant in this range had a stronger inhibitory effect than that of chelators with lower or higher binding constants [2]. In other systems, similar observations are explained by the fact that cytosolic $\mathrm{Ca}^{2+}$ gradients can be most effectively dispersed by chelator with a binding constant in the range of the average $\mathrm{Ca}^{2+}$ concentration in the gradient [33]. It is thus tempting to speculate that a $\mathrm{Ca}^{2+}$-dependent step regulates the fusion event that leads to network formation. A regulatory role for $\mathrm{Ca}^{2+}$ has been established in many other fusion reactions. For example, it controls the fusion of secretory vesicles and of vacuoles [31]. We do not observe the obvious differences of free $\mathrm{Ca}^{2+}$ levels in all the three different ratios of cytosol to vesicles (data not shown).

In a mature Xenopus egg, all of the materials that control membrane breakdown and reassembly are stockpiled in the cell, as shown by the fact that a Xenopus zygote can form over 4000 somatic nuclei [19] during early cell divisions in vitro. The extremely plentiful Xenopus egg can independently and meticulously regulate the disassembly and reassembly of cell structure during early embryonic development in vivo. The components necessary for reassembly of cellular structure are present and stored in Xenopus egg extracts, and thus we can imitate this assembly process by using a cell-free system to better understand the mechanism of cell reconstitution.

\section{Acknowledgments}

We thank Prof Zhuan Zhou (Institute of Molecular Medicine and National Laboratory of Biomembrane and Membrane Biotechnology, Peking University) for providing experimental condition and technique in his laboratory to measure calcium concentration, and Quan Long Lv and Qin Ying Liu for their help in the experimental materials. This work was funded by National Key Basic Research Program of China (No. G1999053905).

\section{References}

1 Rabouille C, Jokitalo E. Golgi apparatues partitioning during cell division (review). Mol Membr Biol 2003; 20:117-127.

2 Dreier L, Rapoport TA. In vitro formation of the endoplasmic reticulum occurs independently of microtubules by a controlled fusion reaction. J Cell Biol 2000; 148:883-898.

3 Lohka MJ, Masui Y. Formation in vitro of sperm pronuclei and mitotic chromosomes induced by amphibian ooplasmic components. Science 1983; 220:719-721.

4 Lu P, Ren M, Zhai ZH. Nuclear reconstitution of plant
(Orychophragmus violaceus) demembranated sperm in cell-free extracts from animal (Xenopus laevis) eggs. J Struct Biol 2001; 136:89-95.

5 Hetzer M, Meyer HH, Walther TC, et al. Distinct AAA-ATPase p97 complex function in discrete steps of nuclear assembly. Nat Cell Biol 2001; 3:1086-1091.

6 Meier E, Miller B, Forbes DJ. Nuclear pore complex assembly studies with a biochemical assay for annulate lamellae formation. J Cell Biol 1995; 29:1459-1472.

7 Lavoie C, Lanoix J, Kan FWK, Paiement J. Cell-free assembly of rough and smooth endoplasmic reticulum. J Cell Sci 1996; 109:1415-1425.

8 Fath KR, Trimbur GM, Burgess DR. Molecular motors and a spectrin matrix associate with Golgi membranes in vitro. J Cell Biol 1997; 139:1169-1181.

9 Ulitzur N, Harel A, Golderg M, Feinstein N, Gruenbaum Y. Nuclear membrane vesicle targeting to chromatin in a Drosophila embryo cell-free system. Mol Biol Cell 1997; 8:1439-1448.

10 Drummond S, Ferrigno P, Lyon C, et al. Temporal differences in the appearance of NEP-B78 and an LBR-like protein during Xenopus nuclear envelope reassembly reflect the ordered recruitment of functionally discrete vesicle types. J Cell Biol 1999; 144:225-240.

11 Collas P. Formation of the sea urchin male pronucleus in cellfree extracts. Mol Reprod Dev 2000; 56:265-270.

12 Bennink ML, Leuba SH, Leno GH, et al. Unfolding individual nucleosomes by stretching single chromatin fibers with optical tweezers. Nat Struct Biol 2001; 8:606-610.

13 Lu P, Zhai ZH. Nuclear assembly of demembranated Xenopus sperm in plant cell-free extracts from Nicotiana ovules. Exp Cell Res 2001; 270:96-101.

14 Harel A, Chan RC, Lachish-Zalait A, et al. Importin $\beta$-negatively regulates nuclear membrane fusion and nuclear pore complex assembly. Mol Biol Cell 2003; 14:4387-4396.

15 Hauri HP, Schweizer A. The endoplasmic reticulum-Golgi intermediate compartment. Curr Opin Cell Biol 1992; 4:600608.

16 Acharya U, McCaffery JM, Jacobs R, Malhotra V. Reconstitution of vesiculated Golgi membrane into stacks of cisternae: requirement of NSF in stack formation. J Cell Biol 1995; 129:577-589.

17 Uchiyama K, Jokitalo E, Lindman M, et al. The localization and phosphorylation of p47 are important for Golgi disassemblyassembly during the cell cycle. J Cell Biol 2003, 161:10671079.

18 Ellenberg J, Siggia ED, Moreira JE, et al. Nuclear membrane dynamics and reassembly in living cells: targeting of an inner nuclear membrane protein in interphase and mitosis. J Cell Biol 1997; 138:1193-1206.

19 Almouzni G, Wolffe AP. Nuclear assembly, structure and function: the use of Xenopus in vitro system. Exp Cell Res 1993; 205:1-15.

20 Rabouille C, Misteli P, Watson R, Warren G. Reassembly of Golgi stacks from mitotic Golgi fragments in a cell-free system. J Cell Biol 1995; 129:605-618.

21 Newport J, Spamm T. Disassembly of the nucleus in mitotic extracts: membrane vesiculation, lamina disassembly, and chromosome condensation are independent process. Cell 1987; 48:219-230. 
22 Sider J R, Mandato C A, Weber K L. Direct observation of microtubule-f-actin interaction in cell free lysates. J Cell Sci 1999; 112:1947-1956.

23 Alberio R, Zakhartchenko V, Motlik J, Wolf E. Mammalian oocyte activation: lessons from the sperm and implications for nuclear transfer. Int J Dev Biol 2001; 45:797-809.

24 Manuel D J, Paiement J. Reconstitution of endoplasmic reticulum in rapidly dividing cells of early Xenopus embryos. Am J Anat 1989; 186:99-113.

25 Perkins G A, Song J Y, Tarsa L, et al. Electron tomography of mitochondria from brown adipocytes reveals crista junctions. J Bioenerg Biomembr 1998; 30:431-442.

26 Becker W M, Kleinsmith L J, Hardin J, eds. The world of the cell, 6th edition. Pearson: Benjamin Cummings, 2006.

27 Seemann J, Pypeart M, Taguchi T, Malsam J, Warren G. Partitioning of the matrix fraction of the Golgi apparatus during mitosis in animal cells. Science 2002; 295:848-851.

28 Colman A, Jones EA, Heasman J. Meiotic maturation in Xenopus oocytes - a link between the cessation of protein secretion and the polarized disappearance of Golgi. J Cell Biol 1985; 101:313318.
29 Warren G. Membrane partitioning during cell division. Annu Rev Biochem 1993; 342:323-348.

30 Misteli T, Warren G. COP-coated vesicles are involved in the mitotic fragmentation of Golgi stacks in a cell-free system. J Cell Biol 1994; 125:269-282.

31 Peters C, Mayer, A. $\mathrm{Ca}^{2+} /$ calmodulin signals the completion of docking and triggers a late step of vacuole fusion. Nature 1998; 396:575-580.

32 Cunningham KW, Fink GR. $\mathrm{Ca}^{2+}$ transport in Saccharomyces cerevisiae. J Exp Biol 1994; 196:57-166.

33 Sullivan KMC, Busa WB, Wilson KL. Calcium mobilization is required for nuclear vesicle fusion in vitro: implications for membrane traffic and $\mathrm{IP}_{3}$ receptor function. Cell 1993; 73:14111422.

34 Busa WB, Nuccitell R. An elevated free calcium wave follows fertilization in eggs of the frog Xenopus laevis. J Cell Biol 1985; 100:1325-1329.

Edited by Thomas D Sargent 